die Straßenproteste, die in den sozialen Netzwerken zunächst an Stärke gewinnen und Unternehmen dazu zwingen, Falschmeldungen aus dem Verkehr zu ziehen oder gegen die Bürger gerichteten Regierungen keine Daten zu verkaufen, so stellt sich die Frage, ob sie sich durch das wachsende Aufkommen von Online-Netzwerken nochmal bestätigen werden: Nachbarschaftsnetzwerke, Kollegengruppen, Freundesgruppen, Migrantencommunitys, nonkonforme Experten, und viele andere? Das Bürgersein lässt sich nicht darauf reduzieren, was die Algorithmen mit uns oder mit den großen Unternehmen anstellen.

\title{
Die einvernehmliche Unterordnung
}

In der jüngsten Phase des Zusammenbruchs der politischen Parteien in Brasilien wurde der Versuch unternommen, Konzepte aus den Sozialwissenschaften neu zu schreiben. Statt das scheinbar irrationale Wählerverhalten zu beurteilen, fragt man sich nach alternativen Wegen, dieses zu verstehen. Der brasilianische Soziologe Carlos Eduardo Martins beschrieb den durch den Sturz Dilma Rousseffs ausgelösten Ausnahmezustand, der im Wahlsieg von Bolsonaro gipfelte, als »faschistisch-liberal«. Er argumentiert, dass diese Verschmelzung zweier scheinbar gegensätzlicher politischer Strömungen sich aktuell dadurch auszeichnet, dass zwar Grundsätze der liberalen Demokratie unter Beibehaltung ihrer formalen Aspekte erhalten bleiben. Die autokratische Machtstruktur, die den politischen Wettbewerb einschränkt und die freie Ausübung der Souveränität oder Proteste durch das Volk verhindert, dringe jedoch weiter vor. Seit dem Wahlkampf in Brasilien, so Martins, beobachten wir den Einsatz begrenzter, aber strategischer Maßnahmen der Gewalt, der Willkür und des Terrors. Sie werden eingesetzt, um Arbeitnehmer- oder Menschenrechtsbewegungen sowie die Opfer des in den Drogenhandel verwickelten Staats zum Schweigen zu bringen. Unterdessen werden neue Bündnisse eingegangen, und zwar zwischen regierungsunfähigen Eliten und transnationalen Megakonzernen, sowie Teilen der Mittel- und Unterschicht, die von der Rezession betroffen sind. Ähnliche Prozesse treten in den letzten Jahren in Argentinien, Mexiko und einigen zentralamerikanischen Ländern auf, wenn auch in kleinerem Maßstab.

Diese Erklärungen stützen die gängigen Argumente bezüglich der Neuformierung der Machtverhältnisse durch die Rechten. Es gibt immer noch keine vollständige Erklärung für die grundlegenden Ursachen dieser massenhaften Zustimmung oder für die Umwandlung der Arbeiterklasse und ande- 
rer Gesellschaftsgruppen, die früher mal Unterstützer der progressiven Parteien waren und inzwischen einen wirtschaftspolitischen Kurs befeuern, der sie selbst zu Verlierern macht. Die Untersuchung der französisch-marokkanischen Journalistin Lamia Oualoulou zeigt weitere Anhaltspunkte auf. Oualoulou untersuchte, wie evangelikale Kirchen sich in den ärmsten Gebieten Brasiliens etablieren. Die katholische Kirche und die Linke hatten sich hingegen zunehmend aus diesen Gebieten zurückgezogen, indem sie sich vor allem auf den städtischen Raum konzentrierten. Der Vormarsch der Evangelikalen und anderer nicht-traditioneller Kirchen hat soziokulturelle und kommunikationsbezogene Ursachen. Zum einen haben sie die Fähigkeit zur Vermittlung von Geborgenheit, Geselligkeit und Schutz für Randgruppen. Darüber hinaus haben sie eigens Medien geschaffen, die sie repräsentieren. Der Staat hat sich aus den Favelas und den benachteiligten Zonen zurückgezogen, während die neoliberale Wirtschaft immer mehr Prekarität produzierte.

Als ich die Leute fragte, warum sie sich evangelikalen Gemeinden angeschlossen hatten, ging es nie um theologische Überzeugungen. Ein Zentraler Treffpunkt sei hingegen das Cemeindehaus: Hier könne man gemeinsam singen, Freunde finden, und die Kinder betreuen lassen. Weder der Staat noch die öffentlichen Einrichtungen für Cesundheit, Arbeit und Bildung, noch die katholische Kirche, sondern die Evangelikalen sind diejenigen, die diese Dienste in vielen Fällen anbieten. Die Menschen sehen in den Cemeindehäusern einen Ort der sozialen Unterstützung. Verliert jemand beispielsweise seine Arbeitsstelle, so findet er über die Cemeinde und deren aktive Mithilfe schnell eine neue. Jemand hat nicht genug zu essen? In der Gemeindeküche gibt es reichlich Reis für alle. Die Evangelikalen nehmen in Brasilien den Raum des Staates ein, und sie nutzen die sich daraus ergebenden kulturellen und politischen Auswirkungen für ihre Zwecke. Die Menschen leben die ganze Zeit über eingesperrt in ihrer kleinen Welt. Das liegt teilweise auch daran, dass progressive Bewegungen, also linke Parteien, diese Menschen unter beträchtlichen Vorurteilen alleine gelassen haben. Aber wir dürfen die engen Verbindungen der Linken mit der katholischen Kirche dabei nicht außer Acht lassen. (Febbro 2018)

Eine weitere Strategie dieser Gruppen besteht in der Schaffung eines Mediensystems, das sie repräsentiert. Es nimmt vernachlässigte Bevölkerungsgruppen wieder neu in den Diskurs auf und unterstützt sie öffentlich. Der zweite Fernsehsender in Brasilien gehört dem Bischof der Universalkirche Edir Macedo, der zusätzlich zu biblischen Botschaften und spirituellen Tröstungen 
Medikamente anbietet, Volksapotheken plant und alternative Bildungsangebote stellt.

Macedo stellte seinen Fernsehsender auch in den Dienst der Bewegung um den Ex-Militär Jair Bolsonaro. In der letzten offiziellen Fernsehdebatte vor der Wahl war Bolsonaro der einzige Kandidat, der seine Teilnahme absagte. Stattdessen strahlte das evangelikale Fernsehen zur exakt gleichen Sendezeit ein exklusives Interview mit ihm aus. Millionen von armen Brasilianern, die der evangelikalen Bewegung angehören, lernen in den Gemeinden, auf Alkohol zu verzichten und die Arbeit zu ehren. Sie nutzen ausschließlich evangelikale Radio- und Fernsehsender und gehören auf Facebook und WhatsApp religiösen Gruppen an. Im katholischsten Land der Welt, das als Wiege der Befreiungstheologie gilt, entwickelt sich nun eine »Theologie des Wohlstands«. Sie propagiert dem Gläubigen, dass

er ein Recht auf alles hat: Auf Cesundheit und auf ein materiell abgesichertes Leben. Und zwar jetzt, und nicht erst im Himmelreich! Fehlt es ihm an etwas, dann nur, weil er nicht weiß, dass es ihm zusteht. So entsteht ein Wandel in der Beziehung zu Cott: Dein Schöpfer muss dir all das geben. Du musst nur wissen, wie du ihn darum bittest. Um Gott danach fragen zu können, musst du unbedingt Teil der evangelikalen Kirche sein, bezahlen und beten. Interessant ist, dass diese Strategie am Ende aufgeht: Wenn Evangelikale dem Cebot, mit dem Trinken aufzuhören und die Arbeit zu ehren, folgen, arbeiten sie härter und trinken weniger. Die Leute erkennen den positiven Einfluss auf ihr Leben, weil sie weniger Geld für Alkohol oder andere Vergnügen ausgeben. Am Ende bleibt mehr Erspartes übrig. Der Pastor verspricht ihnen noch viel mehr, aber die Cläubigen passen ihre Bedürfnisse an die Grundidee an. Die Versprechen des Geistlichen, eines Tages stolzer Besitzer eines Cadillacs zu sein, verwandeln sich in Hoffnungen, zumindest eine besser bezahlte Arbeit oder ein gutes Immobiliendarlehen zu erhalten. Die Leute folgen der Ideologie, selbst wenn dabei nur ein Minimum für sie herausspringt. (Febbro 2018)

Das Beispiel der evangelikalen Kirche soll keinen Versuch darstellen, die Krise in der Beziehung zwischen Staat, Gesellschaft und Bürgern oder den abrupten Übergang zum Bolsonaro-Regime alleinig durch sie zu erklären. Gleichermaßen bieten die Ausführungen über die französischen Gelbwesten keine umfassende Interpretation der dekadenten Repräsentativität des politischen Systems in Frankreich. Sie sind lediglich ein Hinweis auf die Notwendigkeit einer mehrdimensionalen Betrachtung, der zunächst nur zur Untersuchung 
der Rekonfiguration verschiedener Lebensrealitäten und Erfahrungen dient, die in der politischen Soziologie nicht erfasst werden. In der Regel fallen diese unter Begriffe wie »öffentliche Meinung« oder werden unter dem Begriff»Demokratieerziehung « zusammengefasst. Über unsere Betrachtungsweise werden die heterogenen Ausgangssituationen und Bedingungen, die bei Wahlen und sozialer Teilhabe mitspielen, deutlich. Es geht darum, die streng vertikale und der Politik dienende Perspektive zu verlassen. Diese wird den neuen religiösen Dynamiken in der Gesellschaft nicht gerecht. Außerdem übersieht sie die Bevorzugung des urbanen Raums gegenüber den vernachlässigten Randgebieten, sowie die allgemeine Präferenz für Ausländer statt Einheimischer. Dabei entgehen ihr auch die neuen Wege des Volkes, sich mitzuteilen und seine Unzufriedenheit symbolisch zum Ausdruck zu bringen. Ziel ist es, den Zerfall der Demokratie aus der Alltagskultur der Bürger, und nicht aus der Perspektive der Elite zu betrachten.

Diese Schwerpunktverlagerung geht von einer Verflechtung zwischen religiösen Institutionen, Parteien und Gewerkschaften im Hinblick auf die Erwartungen und neuen Wege der Sozialisierung aus. Sie geht auf die Frage ein, wie ein Mensch in der heutigen Zeit zum Bürger erzogen wird. In Ländern wie Mexiko und in Mittelamerika zählen die evangelikale Expansion genau wie die angebotenen Dienste krimineller Vereinigungen zu den »sozialen Diensten«. Sie kompensieren das Angebot an der Stelle, wo öffentliche Einrichtungen keine Not erkennen.

Welche soziokulturellen Veränderungen führen zur Normalisierung der Teilhabe so vieler Sektoren an der Korrumpierung armer Menschen? Wie kommt es zur Salonfähigkeit der Mitwirkung bei mafiösen Organisationen, die auf undemokratische Weise ganze Gebiete und soziale Gefüge unter Kontrolle bringen und systematisch öffentliche Güter entwenden, etwa in Form von Überfällen auf Lebensmitteltransporte und Benzinpipelines? Was bringt Jugendliche und junge Erwachsene dazu, sich massiv an riskanten Kriminalverbrechen zu beteiligen, ihr Leben einem Dasein als Mörder zu widmen oder beim Verstecken tausender Leichen mitzuhelfen? Die weitverbreitete Akzeptanz dieser zerstörerischen Verhaltensweisen in Bezug auf das Soziale erfordert die Erweiterung des Begriffs der Banalität des Bösen nach Hannah Arendt: Die Bevölkerung wird nicht nur durch die grausame Natur des Totalitarismus zum Schweigen gebracht, indem sich dieser ausgehend von der Staatsmacht durchsetzt. Vielmehr ist es seine Ausbreitung in die alltäglichen sozialen Beziehungen, die nicht unter dem Schutz von Gesetzen, Prinzipien und liberalen Organisationsstrukturen stehen. In manchen Fällen sind die 
öffentlichen Institutionen selbst an dieser Zersetzung beteiligt. Dies wurde zum Beispiel in einem Zugeständnis des mexikanischen Präsidenten López Obrador deutlich. Der mexikanische Präsident erklärte, dass Arbeiter und Beamte der staatlichen Firma Pemex zu $80 \%$ Schuld am Treibstoffdiebstahl und dessen illegalen Handel trugen.

Ebenso beobachten wir in Lateinamerika einen Anstieg von Menschenrechtsorganisationen, Antikorruptionsverbänden, und Zusammenschlüssen von Familienmitgliedern Verschwundener, sowie von Studierendenorganisationen und Angestelltenverbänden, die deren Anliegen unterstützen (Dutrénit 2017). Erste neue Erfahrungen mit der Solidarwirtschaft werden in den mittleren und unteren Bevölkerungsschichten ausprobiert; Tauschhandel und soziale Währungen, Selbstverteidigung und gemeinschaftlich organisierte Sicherheitskontrollen durch Anwohner sind ein Versuch, die abwesende Rolle des Staates neu zu besetzen. Gleichzeitig verändern sie jeweils den Sozialvertrag und die Ressourcen zum Überleben.

Auf der anderen Seite nehmen auch autoritäre Antworten auf die täglichen Umwälzungen und für den Umgang mit ihnen zu. Diese Bewegungen zur einvernehmlichen Unterwerfung sind der Grund für den Stimmenzuwachs für eine rechtsgerichtete Wirtschaftspolitik, Fremdenfeindlichkeit, internalisierten Rassismus und für eine Übereinkunft zur Militarisierung vieler Lebensbereiche, die durch den sozialen Zusammenbruch der Wirtschaft auf demokratischem Wege scheinbar unregierbar geworden sind. Wir haben es hierbei nicht mit isolierten Entscheidungen einzelner rational Denkender zu tun. Eher handelt es sich um soziale Vereinbarungen, bei denen kollektive Vorstellungen mobilisiert und als Gesamtsystem vorangetrieben werden. Diesen Prozess als Zumutung durch die Medien und die Eliten oder gar als populistischen Verführungsversuch zu verstehen wäre daher trügerisch und keineswegs zielführend, und es würde auch nichts bringen, der Entwicklung entgegenzuwirken, indem man an vermeintlich vernünftige Menschen appelliert, das demokratische Zusammenleben zu retten. Ein solcher Ansatz wäre umso wirkungsloser in Zeiten, in denen die Verschlechterung der Lebensqualität die meisten Menschen dazu verführt, solche Herausforderungen eher als Frage des Über-statt des Zusammenlebens zu betrachten.

An dieser Stelle möchte ich die konzeptuelle Sichtweise des Sozialtheoretikers David Harvey erweitern, wenn er von der »Akkumulation durch Enteignung« (Harvey 2005) spricht. Mit dem Begriff wird die Enteignung im Sinne des Sprachgebrauchs und der freien Verhaltenswahl der Bürger im Kontext der kulturellen und kommunikativen Logik der neoliberalen Wirtschaft analy- 
siert. Welche neuen Formen der Ausbeutung (sowohl materiell als auch symbolisch) finden statt, wenn Konsumhandlungen, politische Beteiligung und Zivilgesellschaften von Medienunternehmen und digitalen Servern enteignet werden? Die Thematik wirft noch weitere Fragen auf: Auf welche Weise profitieren prekäre Sektoren aller Art von informellen und illegalen Gruppierungen? Wie wird die zivile politische Kultur nun als Teil einer Alltagskultur konfiguriert, die anders ist als diejenige, die der liberalen Konzeption der Staatsbürgerschaft einst als Inspiration diente?

Doch diese letzte Frage scheint mir weiterhin zu sehr in der Moderne verfangen zu sein. Stattdessen möchte ich ein besseres Verständnis für die Verbindungen zwischen der Parapolitik der Parteien und den kriminellen Organisationen, der Infrapolitik der sozialen Bewegungen sowie den unbeständigen und kurzlebigen Aktionen der Medienmächte und der sozialen Netzwerke erlangen. Auf diese Weise konfiguriert sich das (zerbrochene) soziale Gefüge, in dessen Rahmen wir nach einer Möglichkeit suchen müssen, Staatsbürgerschaft wiederherzustellen. 
